\title{
Zwei neue Kombinationstherapien gegen Hepatitis C: Gut, aber nicht gut genug
}

\author{
Beim AASLD-Kongress 2017 vorgestellte neue Studie- \\ nergebnisse zu den HCV-Kombinationstherapien \\ Rusasvir/Uprifosbuvir sowie AL-335/Odalasvir/Sime- \\ previr hatten letztlich zur Folge, dass beide Entwick- \\ lungsprogramme eingestellt wurden.
}

\section{AL-335/Odalasvir/Simeprevir}

Bei der einmal täglich einzunehmenden Dreierkombination AL$335 /$ Odalasvir/Simeprevir ( $800 \mathrm{mg} / 25 \mathrm{mg} / 75 \mathrm{mg}$ ) (JNJ-4178) waren die Hoffnungen auf pangenotypische HCV-Wirksamkeit bereits durch eine beim EASL(European Association for the Study of the Liver)-Kongress 2017 vorgestellte Studie begraben worden. Bei allen anderen Genotypen hoffte man auf eine Therapieverkürzung auf nur sechs Wochen. Dies wäre ein klinischer Vorteil.

In der Phase-Ib-Studie OMEGA-1-Studie waren 365 HCV-Patienten ohne Zirrhose entweder sechs oder acht Wochen lang behandelt worden (Zeuzem S et al. AASLD 2017, Abstract 65). Wie Prof. Stefan Zeuzem von der Universität Frankfurt am Main berichtete, erreichten 97,8\% der Patienten unter achtwöchiger Therapie und 98,9\% unter sechswöchiger Therapie eine anhaltendes virologisches Ansprechen SVR 12. Nur bei fünf Patienten kam es zu einem Rückfall. Vier dieser Rückfälle ereigneten sich bei Infektionen des Genotyps 2c aufgrund von Baseline-Resistenzen. Dies war, neben der bereits bekannten GT3-Schwäche, eine Schwäche zu viel. Die klinische Entwicklung wurde eingestellt, obwohl die Substanz Infektionen der Genotypen 1a und 1b, 2a, $2 \mathrm{~b}, 2 \mathrm{f}, 2 \mathrm{k}, 4$ und $5 \mathrm{zu} 99 \%$ bis $100 \%$ in nur sechs Wochen heilte.

\section{Ruzasvir/Uprifosbuvir}

Ruzasvir/Uprifosbuvir (180 mg/450 mg) ist ein pangenotypischer Kandidat. In der C-BREEZE 2-Studie war die Wirksamkeit der Kombination bei 300 Patienten in zwölfwöchiger Therapie untersucht worden [Lawitz E et al. AASLD 2017, Abstract 61). Bei einer SVR12-Rate von insgesamt $92 \%$ zeigten sich Wirksamkeitseinbrüche bei Patienten mit Genotyp la und insbesondere beim Genotyp 3 (76\%). Es offenbarten sich Probleme mit Baseline-Resistenzen sowie bei Zirrhosepatienten. Insgesamt ist die Wirksamkeit als pangenotypisches Regime suboptimal, berichtete Studienautor Eric Lawitz vom Texas Liver Institute in San Antonio. Der Hersteller stoppte das Programm.

Dr. med. Dirk Einecke

The Liver Meeting 2017, AASLD-Kongress, Washington, 20. - 24.10.2017

\section{Lebertransplantation: Organe HCV-positiver Spender langfristig mit guter Funktion}

\begin{abstract}
Beim „Liver Meeting 2017“ wurden erste Daten zur Lebertransplantation von HCV-positiven Spendern präsentiert. Aufgrund der guten Therapierbarkeit lässt sich der Spenderpool auf diese Weise wohl erweitern.
\end{abstract}

Angesichts des Organmangels könnte die Verwendung von Lebern, deren Spender positiv auf HCV-Antikörper sind, aber keine Anzeichen einer aktiven Infektion aufweisten, den DonorPool erweitern und dazu beitragen, die Anzahl der Patienten auf der Transplantationswarteliste zu verringern, betonte Dr. Khurram Bari, Universität Cincinnati. Er hatte eine Studie mit $25 \mathrm{~Pa}$ tienten vorgestellt und resümierte: Die Verwendung von Leberorganen selektierter HCV-positiver Spender sollte heute erwogen werden, angesichts des moderaten Übertragungsrisikos und der Verfügbarkeit effektiver Therapien. Organe von Spendern mit aktiver HCV-Infektion könnten für Patienten mit aktiver HCV-Infektion auf der Warteliste verwendet werden.

Die 25 Organe von Spendern mit HCV-Antikörpern, aber negativen Tests auf HCV-Nukleinsäure, waren Patienten transplantiert worden, die zum Zeitpunkt des Eingriffes einen negativen HCV-Test aufwiesen. Vier dieser Patienten entwickelten HCVAntikörper in den drei Monaten nach der Transplantation, was einer Transmissionsrate von $16 \%$ entsprechen würde. Allerdings waren die Spender in diesen vier Fällen aktive Drogenkonsumenten, die an Anorexie verstarben. Es lässt sich nicht mit Sicherheit ausschließen, dass die Spender sich kurz vor dem Tod erneut mit $\mathrm{HCV}$ infiziert hatten. Bei einem der Empfänger, der sich infizierte, ließ sich zudem im Nachhinein eruieren, dass er zwei Jahre zuvor eine HCV/HIV-Koninfektion erlitten hatte. Kurzum: Die $16 \%$ ige Transmissionsrate ist mit einem Fragezeichen zu versehen, kommentierte Prof. Christoph Sarrazin vom Leberzentrum Wiesbaden. Auf der anderen Seite ist es aber unerheblich: Patienten, die sich bei der Transplantation mit HCV infizieren, können sicher behandelt werden. Drei der vier Patienten wurden geheilt, der vierte erlag einer Komplikation aus anderer Ursache.

Auch eine Fallserie aus Italien mit elfLebertransplantationen dokumentiert die gute Therapierbarkeit. Sowohl Spender als auch Empfänger waren hier HCV-Antikörper-positiv. Die sich direkt an die Transplantation anschließende DAA-Therapie heilte die Infektionen in allen Fällen aus. Angesichts der guten Behandelbarkeit können Lebern von HCV-positiven Spendern künftig problemlos verwendet werden, resümierte Sarrazin.

Dr. med. Dirk Einecke

The Liver Meeting 2017, AASLD-Kongress, Washington, 20. - 24.10.2017 